\title{
On the Gonality of Metric Graphs
}

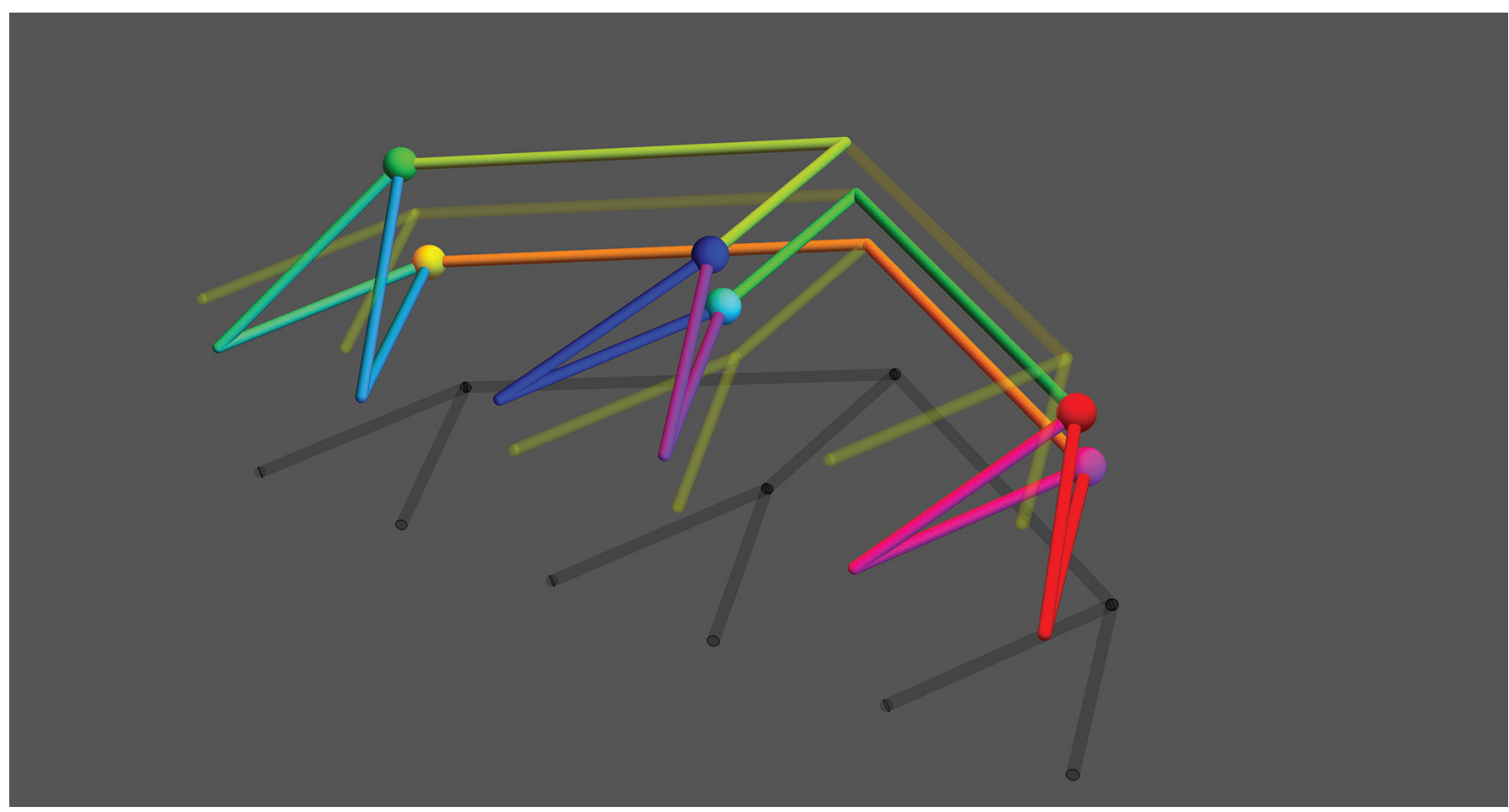

\section{Jan Draisma and Alejandro Vargas}

The last decades have seen an extremely fruitful interplay between Riemann surfaces and graphs with a metric. A deformation process called tropicalisation transforms the former into the latter. Under this process, additional structure on the Riemann surfaces yields additional structure on the metric graphs. For instance, meromorphic functions on Riemann surfaces yield piecewise linear functions on metric graphs. In this manner, theorems in algebraic geometry have deep combinatorial consequences; and conversely, combinatorial arguments can be used to prove theorems in algebraic geometry.

Jan Draisma is a professor of mathematics at the University of Bern and a professor of applied algebra and geometry at Eindhoven University of Technology. His email address is jan.drai sma@math. unibe.ch.

Alejandro Vargas is a PhD student at the University of Bern. His email address is a.vargas.math@gmai 1. com.

Communicated by Notices Associate Editor Steven Sam.

For permission to reprint this article, please contact:

reprint-permission@ams . org.

DOI: https://doi.org/10.1090/noti2277
This interplay has inspired the development of a standalone theory of metric graphs that has strong parallels to the theory of Riemann surfaces. Milestones are a RiemannRoch formula, an Abel-Jacobi theorem, a theory of harmonic morphisms, and a Riemann-Hurwitz formula for metric graphs [BN07, GK08, MZ08, BN09], which closely resemble their classical counterparts for Riemann surfaces.

The purpose of this article is to discuss another beautiful parallel: that of maps where the geometry of the target space is as simple as possible. We summarise the story for Riemann surfaces in Section 1, and for metric graphs in Section 2. In both settings, the number of preimages of any point of the target space, counted with multiplicities, is a constant called the degree of the map. The minimal degree of such a map is called the gonality of the source space. This is a rough measure of the complexity of the space.

The gonality of a Riemann surface is bounded from above by a function of a topological invariant of the Riemann surface: its genus. In groundbreaking work Baker [Bak08] and Caporaso [Cap14] showed that under 
tropicalisation, the gonality either remains constant or decreases. This leads to a similar topological upper bound for the gonality of a metric graph. In Section 3 we outline how such a tropicalisation process works, and how it interacts with the gonality.

The upper bound on the gonality of metric graphs is a purely combinatorial statement whose proof, up to now, depended on algebraic geometry. We have recently completed a purely combinatorial construction of maps witnessing the gonality bound [DV21, Var20]. The technical details of our construction are somewhat daunting-and indeed, we hope that this exposition might incite interest that leads to a simplification!- -but the key ideas are very simple, and we discuss these in Section 4.

While the notion of gonality for metric graphs was inspired by algebraic geometry, it is related to the purely combinatorial graph notion of treewidth and to spectral graph theory; we briefly review these connections in Section 2.

\section{Gonality of Riemann Surfaces}

Recall that a Riemann surface is a connected complex manifold of complex dimension one, hence real dimension two. We restrict our attention to compact Riemann surfaces. As a topological space, such a surface is uniquely determined by its genus, which counts the holes in the surface.

In this setting, the space with the simplest geometry is the unique Riemann surface of genus 0: the Riemann sphere $\mathbb{P}^{1}=\mathbb{C} \cup\{\infty\}$. Any compact Riemann surface $X$ admits a branched cover to $\mathbb{P}^{1}$, i.e., a holomorphic map $\phi$ that, near any $p \in X$, can be expressed as $z \mapsto z^{e}$ for some choice of local coordinates. The integer $e$ is independent of the choice of local coordinates and is called the ramification index of $\phi$ at $p$. If $e>1$, then $\phi$ is called ramified at $p$. The set of points where $\phi$ is ramified is finite.

For a classical example, consider the elliptic curve $X=$ $\mathbb{C} / \Lambda$, the quotient of $\mathbb{C}$ by a lattice $\Lambda:=\mathbb{Z} 1+\mathbb{Z} \tau$ in $\mathbb{C}$. The Weierstrass $\wp$-function defined as

$$
\wp(z)=\frac{1}{z^{2}}+\sum_{\lambda \in \Lambda \backslash\{0\}}\left(\frac{1}{(z+\lambda)^{2}}-\frac{1}{\lambda^{2}}\right)
$$

turns out to be holomorphic around all $z \notin \Lambda$ and has a pole of order 2 at all $z \in \Lambda$. Furthermore, it is doubly periodic with periods 1 and $\tau$, and hence factors through a meromorphic function $X \rightarrow \mathbb{C}$ of degree 2 with a double pole at 0 . This function extends to a holomorphic map $X \rightarrow \mathbb{P}^{1}$ with four index-two ramification points: the points $\{0,1 / 2, \tau / 2,1 / 2+\tau / 2\}+\Lambda$.

The gonality of a compact Riemann surface $X$ is the lowest degree of a surjective holomorphic map from $X$ onto $\mathbb{P}^{1}$. A Riemann surface $X$ has gonality 1 if and only if it is isomorphic to $\mathbb{P}^{1}$, and the gonality of any elliptic curve is 2 . The fundamental relation between gonality and genus is the following.

Theorem 1. The gonality of a compact Riemann surface $X$ of genus $g$ is at most $1+\lceil g / 2\rceil$, with equality if $X$ is sufficiently general. Moreover, if $g$ is even and $X$ is sufficiently general, then the number of holomorphic maps to $\mathbb{P}^{1}$ of degree $1+\mathrm{g} / 2$ from $X$ to $\mathbb{P}^{1}$, counted modulo automorphisms of $\mathbb{P}^{1}$, equals the $\mathrm{g} / 2$ th Catalan number $C_{\mathrm{g} / 2}$.

Compact genus-g Riemann surfaces are parameterised by a complex algebraic variety of dimension $3 g-3$, called the moduli space of genus-g Riemann surfaces, and the predicate "sufficiently general" in Theorem 1 means "for all $X$ in an open dense subset of the moduli space." In fact, a genus- $g$ Riemann surface can have gonality much smaller than $1+\lceil g / 2\rceil$. For example, for every $g \geq 1$ there exist gonality-2 Riemann surfaces of genus $g$. These so-called hyperelliptic curves can be obtained by compactifying the solution set in $\mathbb{C}^{2}$ of an equation $y^{2}=f(x)$, with $f$ a squarefree polynomial of degree $2 g+1$ or $2 g+2$. By Theorem 1 , hyperelliptic curves are quite atypical in the moduli space.

The Catalan numbers $C_{n}=\frac{1}{n+1}\left(\begin{array}{c}2 n \\ n\end{array}\right)$ appear throughout mathematics. We will use the fact that $C_{n}$ is the number of Dyck paths: sequences $p_{0}, p_{1}, \ldots, p_{2 n}$ in $\mathbb{Z}^{2}$ with $p_{0}=(0,1)$, $p_{2 n}=(2 n, 1), p_{i}-p_{i-1} \in\{(1,1),(1,-1)\}$ ("up" and "down" steps, respectively), such that $p_{i}$ lies strictly above the $x$ axis for all $i$.

Theorem 1 is part of Brill-Noether theory, an area of algebraic geometry that has its roots in the late 19th century and is still very active today. The existence of branched covers of the given degree was already established by Riemann, and this was later generalised by Kempf [Kem71] and Kleiman-Laksov [KL72]. The Catalan count was established, in the more general setting of Brill-Noether theory, by Griffiths-Harris [GH80]. Moreover, by [EH87], when $g$ is even, there exists an irreducible variety mapping onto the moduli space of compact genus- $g$ Riemann surfaces whose fibre over a sufficiently general Riemann surface consists of $C_{g / 2}$ points corresponding to the holomorphic maps of that surface onto $\mathbb{P}^{1}$. Our construction sketched in Section 4 yields a metric-graph analogue of this variety.

\section{Gonality of Metric Graphs}

Now we introduce metric graphs and their maps. Let $G=(V, E)$ be a finite, connected, undirected graph; multiple edges and loops are allowed, and we require that $G$ has at least one edge. For every $e \in E$ choose a length $\ell(e) \in \mathbb{R}_{>0}$. Construct a topological space by taking a real interval $I_{e}:=[0, \ell(e)]$ for each $e \in E$ and glueing these together in the manner prescribed by $G$. Equipped with the shortest-path metric, this topological space is what we call a metric graph $\Gamma$. We call the pair $(G, \ell)$ a model for $\Gamma$. Distinct models may yield isometric metric graphs. 


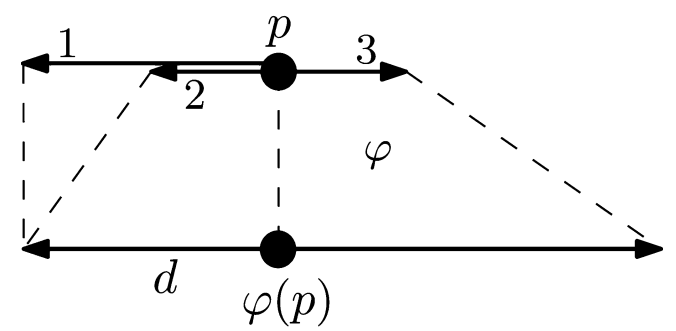

Figure 1. The map $\phi$ is harmonic near $p$. The numbers indicate slopes and we have $m_{\phi}(p)=3$.

Every point $p$ in $\Gamma$ has a neighbourhood isometric to a star of $k$ line segments glued along a common center, where the isometry maps $p$ to the center of the star. The number $k$ is called the valency $\operatorname{val}(p)$ of $p$, and the line segments are called the directions from $p$.

A harmonic map $\phi$ from $\Gamma$ to another metric graph $\Sigma$ is a continuous map that, outside a finite number of points in $\Gamma$, is linear with nonzero integral slopes with respect to the metrics on $\Gamma$ and $\Sigma$, and which satisfies the following balancing condition: for each $p \in \Gamma$ and each direction $d$ from $\phi(p)$, the sum of the slopes of $\phi$ along all directions from $p$ that map onto $d$ is independent of $d$-i.e., replacing $d$ by a different direction from $\phi(p)$ yields the same sum of slopes, which we denote $m_{\phi}(p)$. See Figure 1 for an illustration of the balancing condition and see Figures 2 and 3 for examples of harmonic maps.

We will see in Section 3 that harmonic maps between metric graphs arise as limits of holomorphic maps between Riemann surfaces. For now, a more direct motivation for the definition is as follows: for each real-valued function $f$ defined in an open neighbourhood $U$ of $\phi(p)$ that satisfies the mean-value property, i.e., whose average over the points at distance $\epsilon$ from $q \in U$ equals $f(q)$ for any sufficiently small $\epsilon>0$, the pullback $f \circ \phi$ also satisfies the mean-value property.

By an iterative application of the balancing condition, one finds that $\phi$ is surjective and that, moreover, the number

$$
\sum_{p \in \Gamma: \phi(p)=q} m_{\phi}(p)
$$

is the same for all $q \in \Sigma$. This number is called the degree $d_{\phi}$ of $\phi$. The idea that balancing implies surjectivity with fibres of equal total multiplicity will reappear in a higherdimensional setting in Section 4 .

To tighten the analogy with holomorphic maps even further, one introduces the Riemann-Hurwitz inequality, which requires that at each $p \in \Gamma$ the harmonic map $\phi$ satisfies the condition

$$
\operatorname{val}(p)-2 \geq m_{\phi}(p) \cdot(\operatorname{val}(\phi(p))-2) .
$$

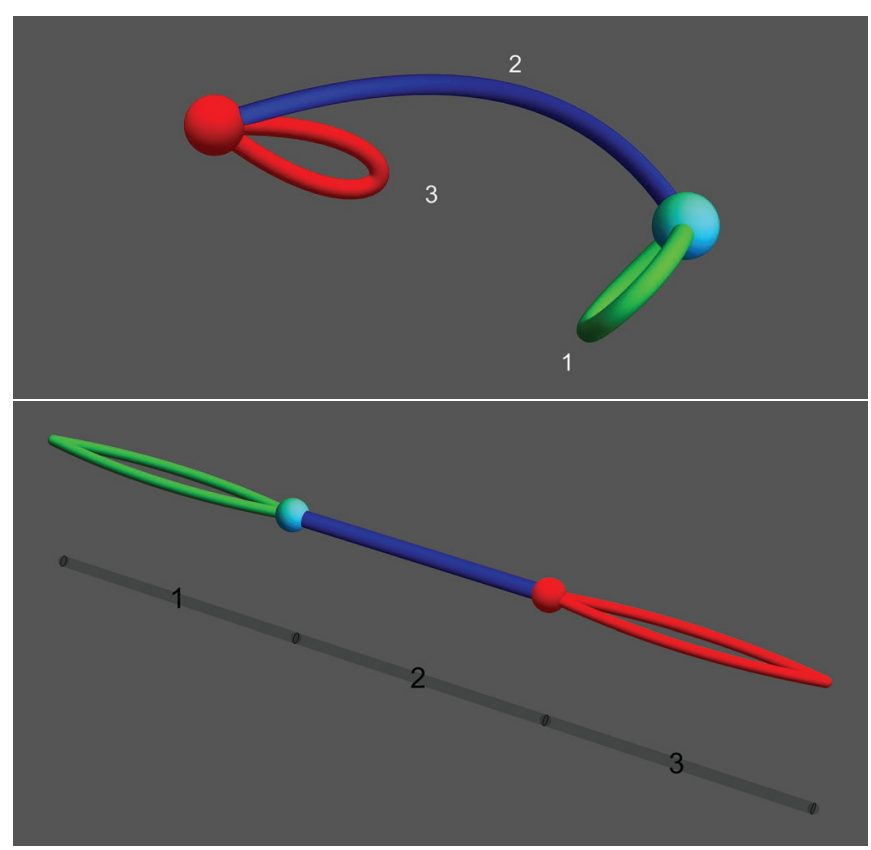

Figure 2. Top: a metric graph of genus 2 called the double lollipop. Bottom: a picture suggesting a tropical morphism of degree 2 from that graph to a line segment. The edge and vertex colours match, the slope is 1 on the red edge and the green edge, and the slope is 2 on the blue edge; to illustrate this, that edge is depicted fatter in the picture below. These slopes ensure balancing at the light-blue and red vertices. The black numbers label edges of the tree below (a path with three edges), the white numbers label edges of the metric graph above.

The origin of this inequality is that the left-hand side minus the right-hand side plus one is the tropical analogue of the ramification index of $\phi$ at $p$, which should, of course, be positive. Note that at all but finitely many points $p$, both sides are equal to zero, and hence $\phi$ has ramification index 1 there. A harmonic map $\phi$ that satisfies the Riemann-Hurwitz inequality at every point $p$ is called a tropical morphism. The map in Figure 2 is a tropical morphism-e.g., in the red vertex the inequality reads $3-2 \geq 2 \cdot(2-2)$-and so is the map in Figure 3 .

Like compact Riemann surfaces, the metric graph $\Gamma$ has a genus, defined as the first Betti number of $\Gamma$, which equals $|E|-|V|+1$ in any model $(G, \ell)$ of $\Gamma$ with $G=(V, E)$. The simplest metric graphs are those of genus 0 . They are the metric trees and play the role of $\mathbb{P}^{1}$. We now introduce an equivalence relation under which all metric trees turn out to be equivalent, so that we may think of them as different representatives of the tropical analogue of $\mathbb{P}^{1}$.

Pick a point $p$ in $\Gamma$ and attach a new line segment to $\Gamma$ at $p$; the segment has no other points glued to $\Gamma$, and we therefore call it a dangling segment in the new metric graph $\tilde{\Gamma}$. A graph $\tilde{\Gamma}^{\prime}$ that can be obtained from $\Gamma$ by a sequence of operations consisting of attaching and/or removing dangling segments is called a tropical modification 


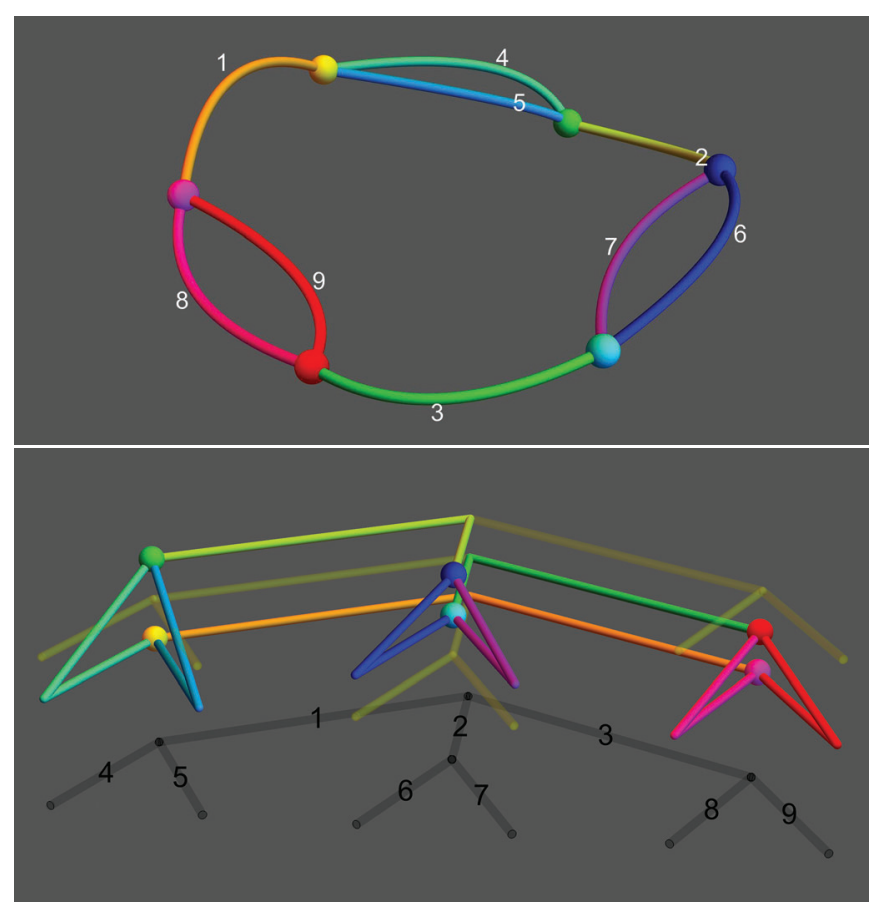

Figure 3. A metric graph of genus 4 on the top, and a tropical morphism of degree 3 from a modification to a tree on the bottom. The edge and vertex colours match, the slope is 1 everywhere, and the dangling edges in the modification are depicted in transparent yellow. The black numbers label edges of the tree below, the white numbers label edges of the metric graph above.

of $\Gamma$. Tropical modification is a ubiquitous operation in tropical geometry, where two different tropicalisations of the same algebraic variety often differ only by a tropical modification.

Every metric graph $\Gamma$ has a tropical modification $\tilde{\Gamma}$ that admits a tropical morphism to a metric tree. Indeed, to construct a tropical morphism to a line segment pick any $p \in \Gamma$ and pin $\Gamma$ to a pinboard with a thumbtack at $p$, letting the rest of $\Gamma$ hang down along a vertical line segment $\Delta=[0, b]$ under the force of gravity. This yields the map $\Gamma \rightarrow \Delta$ that sends each $q \in \Gamma$ to its distance from $p$; it has slope 1 everywhere. By adding suitable dangling edges, this map can be made to satisfy the balancing condition, as well as the Riemann-Hurwitz inequality; see Figure 4.

The tropical morphism to a tree thus constructed typically does not have the minimal possible degree, but at least ensures that the minimal degree exists. We therefore may define the gonality of $\Gamma$ as

$$
\min \left\{d_{\phi} \mid \phi: \tilde{\Gamma} \rightarrow \Delta\right\} \in \mathbb{Z}_{\geq 0},
$$

where $\tilde{\Gamma}$ ranges over all tropical modifications of $\Gamma, \Delta$ ranges over all metric trees, and $\phi$ ranges over all tropical morphisms from $\tilde{\Gamma}$ to $\Delta$.

The gonality of $\Gamma$ is equal to 1 if and only if $\Gamma$ is a metric tree, and is equal to 2 if and only if a tropical modification

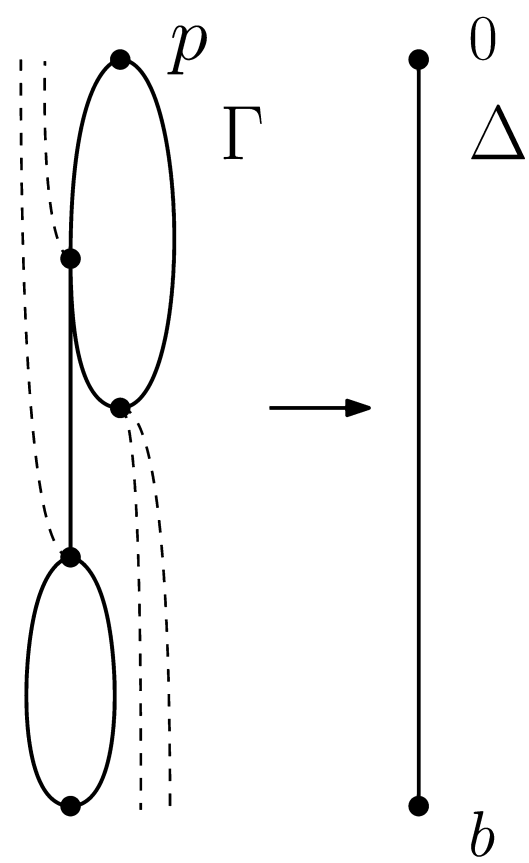

Figure 4. A degree-4 tropical morphism from a modification of the double lollipop graph to a line segment. The dangling segments are dashed.

$\tilde{\Gamma}$ of $\Gamma$ admits an isometric involution $\sigma$ such that $\tilde{\Gamma} / \sigma$ is a metric tree; the moduli space of such tropical hyperelliptic curves is studied in [Cha13].

Over the last years, the gonality of metric graphs has been the subject of research in combinatorics and computer science: the gonality of $\Gamma$ is bounded from below by the treewidth of $G$ for any model $(G, \ell)$ of $\Gamma$ [vDdBG20], and also-like for Riemann surfaces [YY80]-by an expression involving its volume and the smallest positive eigenvalue of the Laplace operator of $\Gamma$ [AK16]. Moreover, like treewidth, gonality is NP-complete [Gij15, BvdWvdZ19].

Figure 3 shows that the genus- 4 graph on the top has gonality at most 3 . This is predicted by the following analogue of Theorem 1.

Theorem 2. The gonality of a metric graph $\Gamma$ of genus $g$ is at most $1+\lceil g / 2\rceil$, with equality if $\Gamma$ is sufficiently general. Moreover, if $g$ is even and $\Gamma$ is sufficiently general, then the number of tropical morphisms $\phi: \tilde{\Gamma} \rightarrow \Delta$ of degree $1+\mathrm{g} / 2$, where $\tilde{\Gamma}$ is a tropical modification of $\Gamma$ and $\Delta$ is a metric tree, equals $C_{g / 2}$, when counted up to simultaneous tropical modification of $\tilde{\Gamma}$ and $\Delta$, as well as with certain multiplicities.

Genus- $g$ metric graphs $\Gamma$, up to modifications, are parameterised by a space of real dimension $(3 g-3)$, the moduli space of genus-g metric graphs, and "sufficiently general" means "for all $\Gamma$ in some open dense subset of that space."

For $g \geq 2$, that moduli space is glued from polyhedral cones $\mathbb{R}_{>0}^{E(G)}$ of dimension $\leq 3 g-3$, one for each genus- $g$ graph $G=(V(G), E(G))$ in which all vertices have valency at least 3; see [Cap12], where a genus- $g$ metric graph is 
called a pure tropical curve. As we move a vector $\ell$ through the cone $\mathbb{R}_{>0}^{E(G)}$, the metric graph with model $(G, \ell)$ moves through the moduli space.

The existence in Theorem 2 of a tropical morphism of degree $1+\lceil g / 2\rceil$ to a tree from some modification of $\Gamma$ is due to Caporaso [Cap14], following work by Baker on the existence of special divisors on metric graphs [Bak08]. They deduce this from Theorem 1 via a degeneration process, a variant of which we review in the next section. The fact that a sufficiently general genus-g metric graph has gonality equal to $1+\lceil g / 2\rceil$ follows from their work combined with work by Cools-Draisma, who, following ideas from Grigory Mikhalkin, do a careful parameter count in [CD18]. In Section 4, we sketch our new contribution to this area: a purely combinatorial proof of Theorem 2, which comes with the bonus of the Catalan count.

\section{Tropicalisation}

In this section, we sketch how Theorem 1 implies part of Theorem 2; more precisely, how the existence of holomorphic maps $X \rightarrow \mathbb{P}^{1}$ of degree $1+\lceil g / 2\rceil$ for compact Riemann surfaces of genus $g$ implies the existence of a tropical morphism $\tilde{\Gamma} \rightarrow \Delta$ from a tropical modification $\tilde{\Gamma}$ of any genus-g metric graph $\Gamma$ to some tree $\Delta$. Instead of following the more algebro-geometric approaches in [Bak08, Cap14], we discuss the hyperbolic-geometric approach taken in [Lan20]. Among other things, the latter paper gives a new viewpoint on Mikhalkin's groundbreaking work on phase-tropical curves [Mik06].

First, at the combinatorial level, there is a straightforward reduction to the case of even $g \geq 2$ and to metric graphs $\Gamma$ that only have points of valency 2 or 3 ; we assume this throughout the remainder of this paper.

The metric graph $\Gamma$ can be realised as a tropical limit of a family of compact Riemann surfaces that we will construct now; we refer to [Lan20] for details on this notion of convergence. The Riemann surfaces in the family are built up from pairs of pants $P_{2 \alpha, 2 \beta, 2 \gamma}$ as in Figure 5, obtained by glueing two copies of the unique right-angled hyperbolic hexagon with three alternating sides of lengths $\alpha, \beta, \gamma>0$ along the remaining three sides. Such a pair of pants has a canonical complex structure.

Let $\left(G=(V, E), \ell \in \mathbb{R}_{>0}^{E}\right.$ ) be the model of $\Gamma$ in which each $v \in V$ has valency 3 , and let $c(e)>0$ be a positive real number for each $e \in E$; the relation between $c$ and $\ell$ will be explained below. Then we construct a compact Riemann surface $X_{c}$ as follows. For each $v \in V$, incident to $e_{1}, e_{2}, e_{3} \in E$, take a copy $P_{v}$ of $P_{c\left(e_{1}\right), c\left(e_{2}\right), c\left(e_{3}\right)}$, and glue these $|V|$ pairs of pants together along their boundary cycles in the manner prescribed by $G$ : if $e=v w \in E$, then $P_{v}$ and $P_{w}$ are glued along their boundary cycle of circumference $c(e)$; if $v=w$ so that $e$ is a loop, then we glue two legs of $P_{v}$. There is one (real) degree of freedom in each

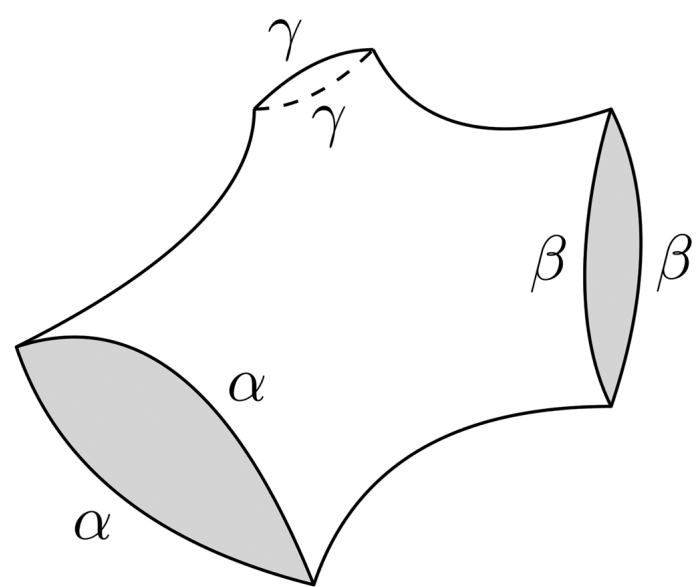

Figure 5. The pair of pants $P_{2 \alpha, 2 \beta, 2 \gamma}$ glued from two geodesic hyperbolic hexagons.

of these $3 g-3$ glueings, namely, the angle under which the cycles are glued. These twists and the cycle lengths are the Fenchel-Nielsen coordinates on Teichmüller space. For our purposes, however, we ignore the twists.

The resulting structure $X_{c}$ inherits the structure of a complex manifold from the hexagons, and is hence a compact Riemann surface. We call $C_{e} \subseteq X_{c}$ the image of the boundary cycle corresponding to $e \in E$.

Now we choose $c$ via the formula

$$
c_{t}(e):=\frac{2 \pi^{2}}{\ell(e) \log (t)} .
$$

Then, as we let $t$ tend to $\infty$, the $c_{t}(e)$ tend to zero and the Riemann surface $X_{t}:=X_{c_{t}}$ tropically converges to the metric graph $\Gamma$ in the sense of [Lan20, Definition 1.1]. This convergence is related to, but different from, convergence in the Gromov-Hausdorff sense; see [Lan20, Remark 3.12].

Next, let $\psi_{t}: X_{t} \rightarrow \mathbb{P}^{1}$ be a holomorphic map of degree $1+g / 2$; these exist by Theorem 1 . We sketch how, in the limit for $t \rightarrow \infty$, these yield a tropical morphism from $\Gamma$ to a tree. For this take $t \gg 0$. Then the boundary cycles $C_{e}$ have disjoint images $\tilde{C}_{e}:=\psi_{t}\left(C_{e}\right) \subseteq \mathbb{P}^{1}, e \in E$. A priori, each $\tilde{C}_{e}$ is only an immersed circle in $\mathbb{P}^{1}$. But it can be shown that after a suitable deformation of the $C_{e}$, their images $\tilde{C}_{e}$ are disjoint, closed circles on the sphere $\mathbb{P}^{1}$; we assume this from now on. Note that each $\tilde{C}_{e}$ may be covered multiple times by $C_{e}$. Make a graph $T$ whose vertices are the connected components of $\mathbb{P}^{1} \backslash \bigcup_{e \in E} \tilde{C}_{e}$ and two are connected by an edge if they have a common $\tilde{C}_{e}$ in their boundary. The fact that $\mathbb{P}^{1}$ has genus 0 implies that $T$ is a tree.

For each $e \in E, \psi_{t}^{-1}\left(\tilde{C}_{e}\right)$ is a disjoint union of circles in $X_{t}$; one of these is $C_{e}$, and the other circles are disjoint from all boundary cycles. Cut up $X_{t}$ along all of these (old and new) circles and construct $G^{\prime}=\left(V^{\prime}, E^{\prime}\right)$ from the connected components of the cut-up $X_{t}$, like we constructed $T$ from the cut-up $\mathbb{P}^{1}$. Then $G^{\prime}=\left(V^{\prime}, E^{\prime}\right)$ is obtained from 
$G$ by subdividing edges and attaching dangling trees. Each edge $e^{\prime} \in E^{\prime}$ corresponds to a circle $C_{e^{\prime}}$ in $X_{t}$ mapping to some circle $\tilde{C}_{e^{\prime}}$ l let $m_{e^{\prime}} \in \mathbb{Z}_{\geq 1}$ be the topological degree of the restriction $\left.\psi_{t}\right|_{C_{e^{\prime}}}: C_{e^{\prime}} \rightarrow \tilde{C}_{e}$.

The formula for $c_{t}(e)$ in (1) guarantees that $\psi_{t}$ tropically converges to a tropical morphism $\phi$ from a modification $\Gamma^{\prime}$ of $\Gamma$, with underlying graph $G^{\prime}$, to the metric tree $\left(T, \ell_{T}\right)$ for a suitable edge length vector $\ell_{T}$ [Lan20]. The tropical morphism $\phi$ is linear on the open line segment in $\Gamma$ corresponding to an edge $e^{\prime}$ in $G^{\prime}$, with slope equal to $m_{e^{\prime}}$. In particular, the balancing condition and the RiemannHurwitz condition are immediate here.

On the other hand, if a point $p$ in $\Gamma^{\prime}$ corresponds to a vertex $v^{\prime} \in V^{\prime}$ of $G^{\prime}$ and hence to a connected component $U$ of $X_{t} \backslash \bigcup_{e^{\prime} \in E^{\prime}} C_{e^{\prime}}$, then the balancing condition and the Riemann-Hurwitz inequality at $p$ follow from the fact that the restriction $\left.\psi_{t}\right|_{\bar{U}}: \bar{U} \rightarrow \psi_{t}(\bar{U})$ is a branched cover between Riemann surfaces with boundary.

This concludes our discussion of the relation between Theorems 1 and Theorem 2.

\section{Proof Sketch of Theorem 2}

In this section, we sketch our new, purely combinatorial proof of Theorem 2. As in the previous section, we assume that $g$ is even and that all points in $\Gamma$ have valency 2 or 3 .

Full-dimensional families of morphisms. Consider a triple $(\Gamma, \Delta, \phi: \tilde{\Gamma} \rightarrow \Delta)$ of a trivalent genus-g metric graph $\Gamma$, a metric tree $\Delta$, and a tropical morphism $\phi$ : $\tilde{\Gamma} \rightarrow \Delta$, where $\tilde{\Gamma}$ is a tropical modification of $\Gamma$. Let ( $G=(V(G), E(G)), \ell_{\Gamma} \in \mathbb{R}_{>0}^{E(G)}$ ) be the model of $\Gamma$ for which each $v \in V(G)$ has valency 3 . We will say that points of $\tilde{\Gamma}$ lie above their images under $\phi$ in $\Delta$. The first key idea in our proof is to look at such triples that move in a family of the right dimension, namely, $3 g-3$-recall from Section 2 that this is the dimension of the moduli space of genus- $g$ metric graphs, and equal to $|E(G)|$.

"Moving in a $(3 g-3)$-dimensional family" is to be understood as follows. Call $p \in \Delta$ ordinary if $p$ has valency 2 in $\Delta$ and a sufficiently small open interval $I$ around $p$ in $\Delta$ has the property that $\phi^{-1}(I)$ is a disjoint union of open intervals, each mapped bijectively onto $I$ with some integer slope; and call $p$ special otherwise. The special points are those above which the combinatorics of $\phi$ changes. If we delete from $\Delta$ all special points $p$, then our first requirement is that $\Delta$ decomposes into $3 g-3$ open intervals. We write $E(T)$ for the set of these intervals; $E(T)$ is the edge set of a tree $T$, a model of $\Delta$, and we let $\ell_{\Delta} \in \mathbb{R}_{>0}^{E(T)}$ be the edge length vector for the metric graph $\Delta$.

Now each edge in $\Gamma$ passes above one or more of the edges in $\Delta$, possibly multiple times and with different slopes. Consequently, each edge length in $\Gamma$ is a nonnegative rational linear combination of the edge lengths in $\Delta$. We record the coefficients of these combinations in

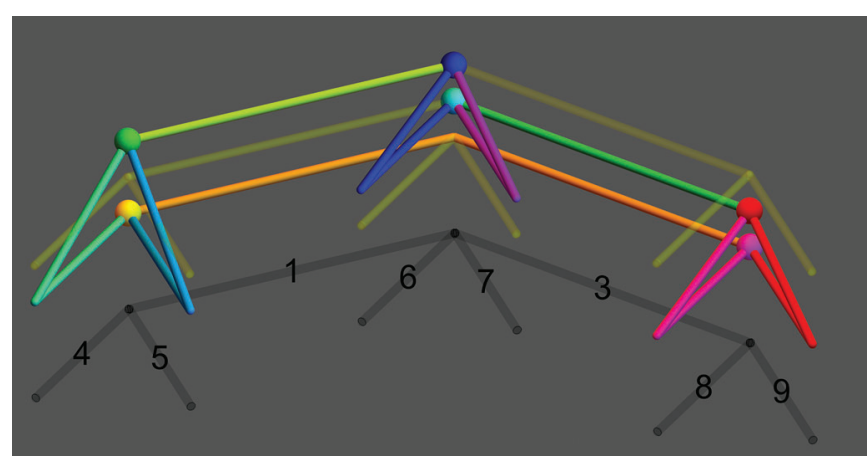

Figure 6. The edge labelled 2 in the tree in Figure 3 has been contracted to obtain $\phi_{0}$.

an $E(G) \times E(T)$-matrix $A_{\phi}$, so that $\ell_{\Gamma}=A_{\phi} \ell_{\Delta}$. Our second requirement is that $A_{\phi}$ is a nonsingular matrix. If both requirements are fulfilled, then we say that the triple moves in a family of the right dimension $3 g-3$.

For instance, in Figures 2 and 3, the matrix $A_{\phi}$ equals

$$
\left[\begin{array}{ccc}
2 & 0 & 0 \\
0 & 1 / 2 & 0 \\
0 & 0 & 2
\end{array}\right],\left[\begin{array}{lllllllll}
1 & 0 & 1 & 0 & 0 & 0 & 0 & 0 & 0 \\
1 & 1 & 0 & 0 & 0 & 0 & 0 & 0 & 0 \\
0 & 1 & 1 & 0 & 0 & 0 & 0 & 0 & 0 \\
0 & 0 & 0 & 2 & 0 & 0 & 0 & 0 & 0 \\
0 & 0 & 0 & 0 & 2 & 0 & 0 & 0 & 0 \\
0 & 0 & 0 & 0 & 0 & 2 & 0 & 0 & 0 \\
0 & 0 & 0 & 0 & 0 & 0 & 2 & 0 & 0 \\
0 & 0 & 0 & 0 & 0 & 0 & 0 & 2 & 0 \\
0 & 0 & 0 & 0 & 0 & 0 & 0 & 0 & 2
\end{array}\right],
$$

respectively, relative to the labellings of the edges in $G$ (in white) and $T$ (in black) indicated there, and both of these matrices are nonsingular $(3 g-3) \times(3 g-3)$-matrices.

Balancing. As we vary $\ell_{\Delta}$ in the cone $\mathbb{R}_{>0}^{E(T)}$, its image $\ell_{\Gamma}=$ $A_{\phi} \ell_{\Delta}$ moves through an open cone $C_{\phi}$ in $\mathbb{R}_{>0}^{E(G)}$, which maps to an open subset of the moduli space of genus-g graphs. For instance, in Figure 3, one facet of that cone is given by the condition

$$
\ell_{\Gamma}\left(e_{2}\right)+\ell_{\Gamma}\left(e_{3}\right)-\ell_{\Gamma}\left(e_{1}\right)>0 .
$$

This expresses that, for this combinatorial type of tropical morphisms, the orange edge with the white label 1, which is mapped onto the edges of the tree with the black labels 1 and 3, has length less than the sum of the lengths of the yellow edge (white label 2, mapped onto black 1 and 2) and the green edge (white label 3, mapped onto black 2 and 3).

The second key ingredient in our proof is a careful analysis of what happens when a single entry of $\ell_{\Delta}$ becomes zero. Call the resulting vector $\ell_{\Delta_{0}}$ and set $\ell_{\Gamma_{0}}:=A_{\phi} \ell_{\Delta_{0}}$ and let $\phi_{0}: \tilde{\Gamma}_{0} \rightarrow \Delta_{0}$ be the corresponding tropical morphism. See Figure 6.

If the nonzero entries of $\ell_{\Delta_{0}}$ are sufficiently general, then there are two possibilities: either $\ell_{\Gamma_{0}}$ has only positive entries, or precisely one entry is zero. In the former case, 


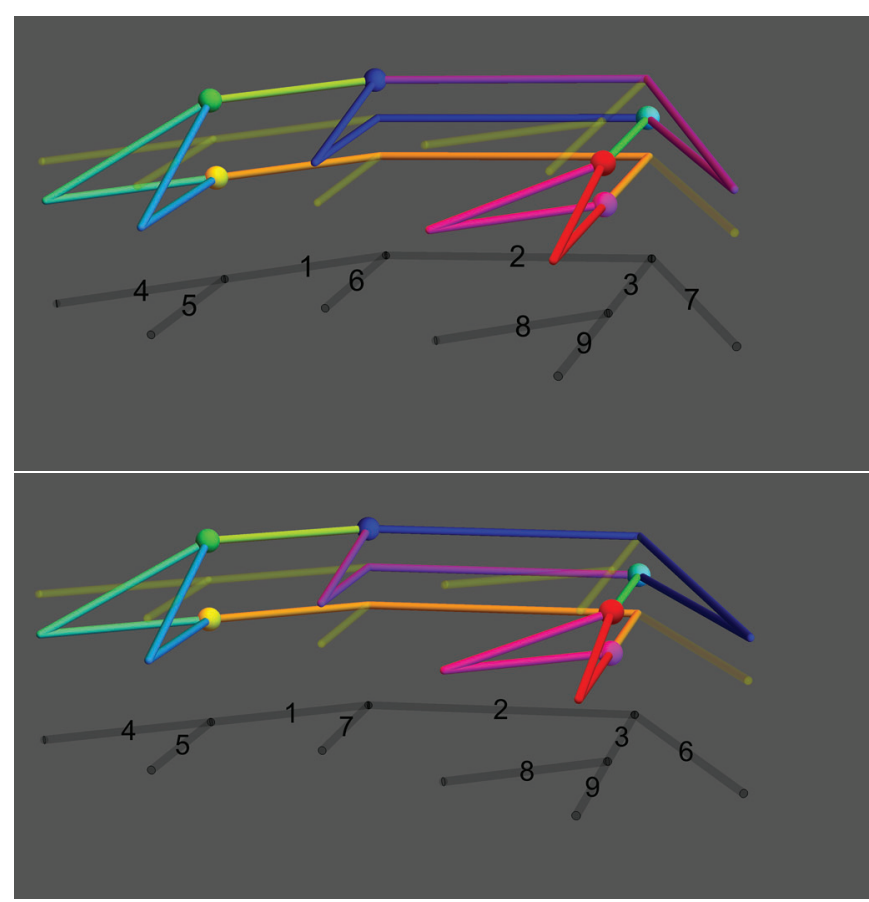

Figure 7. $\phi_{2}$ and $\phi_{3}$ with $\phi_{0}$ as limit upon contracting the edge labelled 2 .

the metric graph $\Gamma_{0}$ still has the same (trivalent) combinatorial structure as $\Gamma$-this is what happens in Figure 6while in the latter case, $\Gamma_{0}$ has precisely one vertex of valency 4.

In either case, we construct all combinatorial types of tropical morphisms $\phi_{1}, \ldots, \phi_{k}$, with $\phi_{1}$ equal to $\phi$, that have $\phi_{0}$ as a limit when shrinking one edge of the underlying tree with $3 g-3$ edges. This can be done since all the axioms for a tropical morphism can be checked locally, so we need only to study local changes in the combinatorics of $\phi_{0}$ near the vertex corresponding to the shrunk edge. The matrices $A_{\phi_{l}}$ are all equal to $A_{\phi}$ except in the column corresponding to the edge of the tree that needs to be shrunk to arrive at $\phi_{0}$.

Ideally, from among the $\phi_{l}$, we would like to keep only those that move in a family of the right dimension. However, in the first case, where $\Gamma_{0}$ is still trivalent, this cannot be done via local combinatorial arguments: the nonsingularity of the matrix $A_{\phi_{l}}$ depends on the global structure of the morphism $\phi_{l}$. However, it turns out that this is irrelevant for our approach: using local combinatorial arguments and the fact that the matrices $A_{\phi_{l}}$ all agree outside one column, we show that there exist positive rational numbers $q_{1}, \ldots, q_{k}>0$ such that the balancing equation

$$
q_{1} \operatorname{det}\left(A_{\phi_{1}}\right)+\cdots+q_{k} \operatorname{det}\left(A_{\phi_{k}}\right)=0
$$

holds; a $\phi_{l}$ that does not move in a family of the right dimension contributes zero to this equation. Since $\operatorname{det}\left(A_{\phi_{1}}\right)$ is nonzero, the balancing equation implies that there exists at least one $l>1$ for which $\phi_{l}$ moves in a family of the right dimension, and indeed one for which $\operatorname{det}\left(A_{\phi_{1}}\right)$ and $\operatorname{det}\left(A_{\phi_{l}}\right)$ have opposite signs.

In our running example, $k$ equals 3 and $\phi_{2}, \phi_{3}$ are seen in Figure 7. Furthermore, we have

$$
A_{\phi_{2}}=A_{\phi_{3}}=\left[\begin{array}{lllllllll}
1 & 1 & 1 & 0 & 0 & 0 & 0 & 0 & 0 \\
1 & 0 & 0 & 0 & 0 & 0 & 0 & 0 & 0 \\
0 & 0 & 1 & 0 & 0 & 0 & 0 & 0 & 0 \\
0 & 0 & 0 & 2 & 0 & 0 & 0 & 0 & 0 \\
0 & 0 & 0 & 0 & 2 & 0 & 0 & 0 & 0 \\
0 & 1 & 0 & 0 & 0 & 2 & 0 & 0 & 0 \\
0 & 1 & 0 & 0 & 0 & 0 & 2 & 0 & 0 \\
0 & 0 & 0 & 0 & 0 & 0 & 0 & 2 & 0 \\
0 & 0 & 0 & 0 & 0 & 0 & 0 & 0 & 2
\end{array}\right]
$$

and the balancing equation reads

$$
\begin{aligned}
& 2^{-6} \operatorname{det}\left(A_{\phi_{1}}\right)+2^{-6} \operatorname{det}\left(A_{\phi_{2}}\right)+2^{-6} \operatorname{det}\left(A_{\phi_{3}}\right) \\
& =2+(-1)+(-1)=0 .
\end{aligned}
$$

In this example, the matrices $A_{\phi_{2}}$ and $A_{\phi_{3}}$ turn out to be the same, and indeed the tropical morphisms $\phi_{2}$ and $\phi_{3}$ have the same combinatorics; but they differ when we keep track of the (colours of the) edges of $\Gamma$-blue and purple are swapped-and therefore need to be counted both.

A similar analysis can be done in the second case, where $\Gamma_{0}$ has a four-valent vertex. In that case, around $\Gamma_{0}$ there are precisely three combinatorial types of metric graphs, corresponding to the three different groupings of the four edges into two pairs. Here we find positive rational numbers $q_{i}$ such that $q_{i} \operatorname{det}\left(A_{\phi_{i}}\right)$ is independent of $i$.

Finally, in both cases, it turns out that the numbers $q_{i}$ can be chosen to depend on the morphism $\phi_{i}$ only (not on the limit that we are considering), and such that the $q_{i} \operatorname{det}\left(A_{\phi_{i}}\right)$ are certain integers. We call their absolute value the multiplicity of the morphism $\phi_{i}$. Then our result shows that the sum of the multiplicities of the morphisms $\widetilde{\Gamma^{\prime}} \rightarrow \Delta^{\prime}$ near $\phi_{0}$ is constant (independent of $\Gamma$ ).

In our running example, these multiplicities are 2 for $\phi_{1}$, where (2) holds; and 1 for both $\phi_{2}$ and $\phi_{3}$, where the opposite of (2) holds-so we see that the open cone in $\mathbb{R}_{>0}^{E(G)}$ parameterised by $A_{\phi_{1}}$ is adjacent to, and "balances out," the two (identical) cones parameterised by $A_{\phi_{2}}, A_{\phi_{3}}$.

Just like the balancing condition for a harmonic morphism between metric graphs ensures that the morphism is surjective and has fibres of the same total multiplicity, our higher-dimensional balancing condition implies that each genus-g metric graph admits the same number of degree- $(1+(g / 2))$ tropical morphisms to a tree, when counted with multiplicities. Note that the multiplicities are nonzero only for tropical morphisms that move in a family of the right dimension, so "accidental" tropical morphisms, for instance those from a hyperelliptic graph to a tree, will not be counted here. 


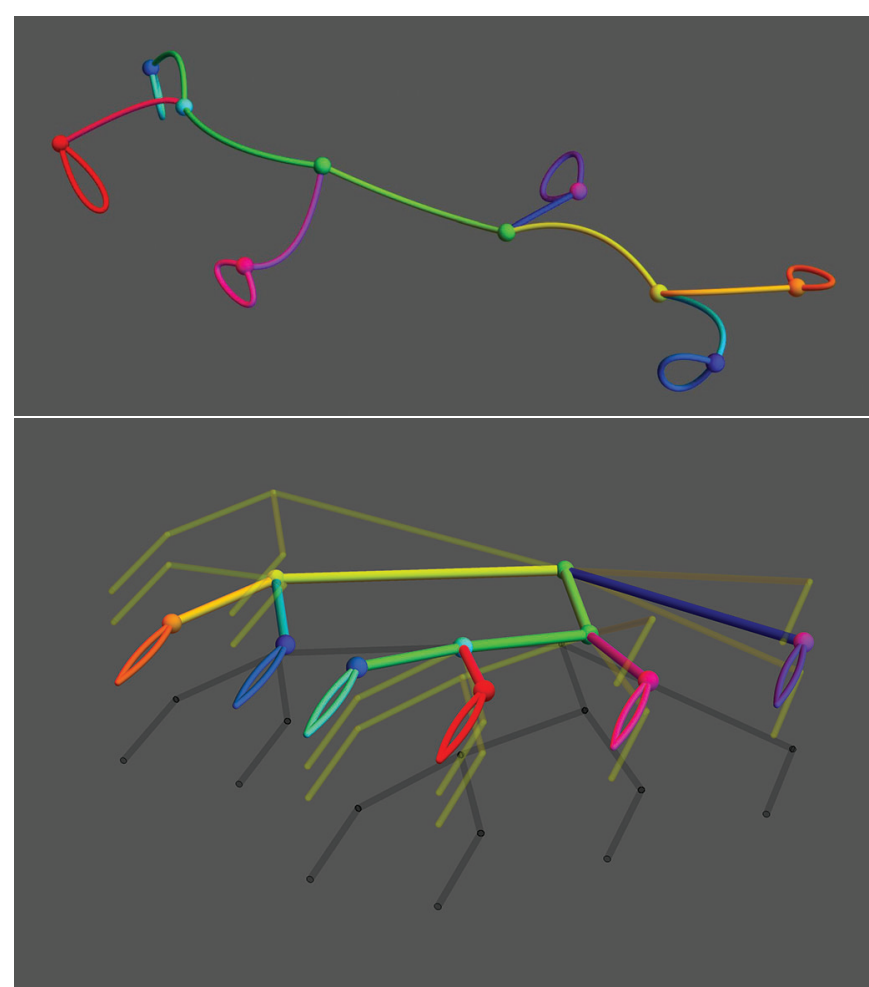

Figure 8. Top: a caterpillar of genus 6 . Bottom: one of its $C_{3}=5$ tropical morphisms to a metric tree.

The two remaining ingredients in our proof of Theorem 2 are: first, that the space of such morphisms $\phi: \tilde{\Gamma} \rightarrow \Delta$ is connected, and second, that the correct count is the Catalan number $C_{g / 2}$. The connectedness follows from two facts: first, that the space of metric graphs of genus $g$ is connected through codimension 1 (indeed, this is true even when restricting to graphs with higher connectivity [Cap12]); and second, an analysis of a special class of graphs discussed in the next paragraph.

Caterpillars of loops. Consider a "caterpillar of $g$ loops": a metric graph $\Gamma_{\mathrm{CL}}$ whose underlying graph $G_{\mathrm{CL}}$ is obtained by taking a path $v_{0}, e_{1}, v_{1}, \ldots, e_{g-1}, v_{g-1}$ of $g-1$ edges and attaching loops to $v_{0}$ and $v_{g-1}$; and lollipops (bridges leading to a loop) to the remaining $v_{i}$. See Figure 8 .

Given a tropical morphism $\phi: \tilde{\Gamma}_{\mathrm{CL}} \rightarrow \Delta$ from a tropical modification of $\Gamma$ to a metric tree, it turns out that $\phi$ is linear on each edge $e_{i}$; let $m_{\phi}\left(e_{i}\right)$ denote its slope. Then we show that the sequence $(0,1),\left(1, m_{\phi}\left(e_{1}\right)\right), \ldots,(g-$ $\left.1, m_{\phi}\left(e_{g-1}\right)\right),(g, 1)$ is a Dyck path. Conversely, given a Dyck path, there is a unique tropical morphism from a modification of $\Gamma_{\mathrm{CL}}$ to a metric tree whose slopes along the edges $e_{i}$ are the second coordinates of the points in the Dyck path.

For instance, in Figure 8, if we let $v_{0}$ be the red vertex and $v_{g-1}=v_{5}$ be the orange vertex, then the slopes along $e_{1}, e_{2}, e_{3}, e_{4}, e_{5}$ are $2,3,4,3,2$, so the Dyck path is $(0,1),(1,2),(2,3),(3,4),(4,3),(5,2),(6,1)$.
The multiplicities of all these tropical morphisms turn out to be 1 . So $\Gamma_{\mathrm{CL}}$ admits precisely $C_{g / 2}$ tropical morphisms from modifications to trees. Furthermore, all $C_{g / 2}$ Dyck paths are connected to each other via moves that change an occurrence of up-down into down-up or vice versa. We show that these moves can be realised via the type of moves on tropical morphisms discussed in the previous subsection. Together with the balancing formula (3), this implies that the space of morphisms from (modifications of) genus- $g$ metric graphs to metric trees that move in a family of dimension $3 g-3$ is connected, and that a general metric graph of genus $g$ has precisely $C_{g / 2}$ such morphisms. This concludes our proof sketch of Theorem 2.

An animation of a random walk through the space of tropical morphisms in the genus- 4 case can be found at https://mathsites.unibe.ch/jdraisma/Movie Genus4.mp4.

\section{Conclusion}

We have given a brief account of classical and recent developments on gonality for Riemann surfaces and metric graphs, with a focus on our new combinatorial proof of Theorem 2.

We conclude this exposition with four questions that we would very much like to see settled:

1. Our proof, while conceptually very simple, requires the analysis of a large number of different cases. Does there exist a more straightforward proof of the balancing equation (3), which is the core of the proof?

2. What is the algebro-geometric interpretation of the multiplicities in the balancing condition?

3. Baker conjectured [Bak08], roughly speaking, that when $\Gamma$ has integral edge lengths, then $\phi: \Gamma^{\prime} \rightarrow \Delta$ can also, in a suitable sense, be chosen integral. Can this be proved or disproved using our methods? Our intuition is that tropical morphisms of higher multiplicity might not always be chosen integral, but we do not have a precise statement to this effect.

4. If $\phi: \tilde{\Gamma} \rightarrow \Delta$ is a degree- $d$ tropical morphism from a modification of a metric graph $\Gamma$ to a tree $\Delta$, then the fibre over any $q \in \Delta$, with each $p \in \phi^{-1}(q)$ counted with multiplicity $m_{\phi}(p)$, is a divisor of rank at least one on $\tilde{\Gamma}$ in the sense of [Bak08], and can be made into a rank-one divisor on $\Gamma$ by replacing all $p$ on dangling trees to the points where those trees are attached to $\Gamma$. So in particular, our theorem gives a combinatorial proof of the theorem from [Bak08] that there exists a divisor on any genus- $g$ metric graph $\Gamma$ of degree equal to $1+\lceil g / 2\rceil$ and rank at least 1 . Does there exist a generalisation of our proof to the existence of higher-rank divisors on metric graphs?

The last question seems very challenging indeed; we are not aware of a combinatorial-geometric notion of 
morphisms from metric graphs into higher-dimensional tropical projective spaces that might facilitate such a generalisation.

ACKNOWLEDGMENTS. We thank Lionel Lang for pointing out an inaccuracy in an earlier version of this expository paper and explaining aspects of his paper [Lan20] to us; the Institut Mittag-Leffler, where some of the research described here was done during their Spring 2018 semester on Tropical Geometry, Amoebas, and Polytopes; and two referees whose suggestions greatly improved our exposition.

Jan Draisma is partially funded by Vici grant 639.033.514 from the Netherlands Organisation for Scientific Research.

\section{References}

[AK16] Omid Amini and Janne Kool, A spectral lower bound for the divisorial gonality of metric graphs, Int. Math. Res. Not. IMRN 8 (2016), 2423-2450, DOI 10.1093/imrn/rnv213. MR3519119

[Bak08] Matthew Baker, Specialization of linear systems from curves to graphs, Algebra Number Theory 2 (2008), no. 6, 613-653, DOI 10.2140/ant.2008.2.613. With an appendix by Brian Conrad. MR2448666

[BN07] Matthew Baker and Serguei Norine, Riemann-Roch and Abel-Jacobi theory on a finite graph, Adv. Math. 215 (2007), no. 2, 766-788, DOI 10.1016/j.aim.2007.04.012. MR2355607

[BN09] Matthew Baker and Serguei Norine, Harmonic morphisms and hyperelliptic graphs, Int. Math. Res. Not. IMRN 15 (2009), 2914-2955, DOI 10.1093/imrn/rnp037. MR2525845

[BvdWvdZ19] Hans L. Bodlaender, Marieke van der Wegen, and Tom C. van der Zanden, Stable divisorial gonality is in NP, SOFSEM 2019: theory and practice of computer science, Lecture Notes in Comput. Sci., vol. 11376, Springer, Cham, 2019, pp. 81-93, DOI 10.1007/978-3-030-108014_8. MR3912327

[Cap12] Lucia Caporaso, Geometry of tropical moduli spaces and linkage of graphs, J. Combin. Theory Ser. A 119 (2012), no. 3, 579-598, DOI 10.1016/j.jcta.2011.11.011. MR2871751

[Cap14] Lucia Caporaso, Gonality of algebraic curves and graphs, Algebraic and complex geometry, Springer Proc. Math. Stat., vol. 71, Springer, Cham, 2014, pp. 77-108, DOI 10.1007/978-3-319-05404-9_4. MR3278571

[Cha13] Melody Chan, Tropical hyperelliptic curves, J. Algebraic Combin. 37 (2013), no. 2, 331-359, DOI 10.1007/s10801-012-0369-x. MR3011346

[CD18] Filip Cools and Jan Draisma, On metric graphs with prescribed gonality, J. Combin. Theory Ser. A 156 (2018), 121, DOI 10.1016/j.jcta.2017.11.017, MR3762100

[DV21] Jan Draisma and Alejandro Vargas, Catalanmany tropical morphisms to trees; Part I: Constructions, J. Symbolic Comput. 104 (2021), 580-629, DOI 10.1016/j.jsc.2020.09.005. MR4180142
[EH87] David Eisenbud and Joe Harris, Irreducibility and monodromy of some families of linear series, Ann. Sci. École Norm. Sup. (4) 20 (1987), no. 1, 65-87. MR892142

[GK08] Andreas Gathmann and Michael Kerber, A RiemannRoch theorem in tropical geometry, Math. Z. 259 (2008), no. 1, 217-230, DOI 10.1007/s00209-007-0222-4. MR2377750

[Gij15] Dion Gijswijt, Computing divisorial gonality is hard, arXiv:1504.06713 (2015).

[GH80] Phillip Griffiths and Joseph Harris, On the variety of special linear systems on a general algebraic curve, Duke Math. J. 47 (1980), no. 1, 233-272. MR563378

[Kem71] G. Kempf, Schubert methods with an application to algebraic curves, Stichting Mathematisch Centrum, 1971.

[KL72] Steven L. Kleiman and Dan Laksov, On the existence of special divisors, Amer. J. Math. 94 (1972), 431-436, DOI 10.2307/2374630. MR323792

[Lan20] Lionel Lang, Harmonic tropical morphisms and approximation, Math. Ann. 377 (2020), no. 1-2, 379-419, DOI 10.1007/s00208-020-01971-0. MR4099633

[Mik06] Grigory Mikhalkin, Tropical geometry and its applications, International Congress of Mathematicians. Vol. II, Eur. Math. Soc., Zürich, 2006, pp. 827-852. MR2275625

[MZ08] Grigory Mikhalkin and Ilia Zharkov, Tropical curves, their Jacobians and theta functions, Curves and abelian varieties, Contemp. Math., vol. 465, Amer. Math. Soc., Providence, RI, 2008, pp. 203-230, DOI 10.1090/conm/465/09104. MR2457739

[vDdBG20] Josse van Dobben de Bruyn and Dion Gijswijt, Treewidth is a lower bound on graph gonality, Algebr. Comb. 3 (2020), no. 4, 941-953, DOI 10.5802/alco. 124 MR4145985

[Var20] Alejandro Vargas, Catalan-many tropical morphisms to trees; Part II: Counting (2020). Manuscript, to appear on arXiv soon.

[YY80] Paul C. Yang and Shing Tung Yau, Eigenvalues of the Laplacian of compact Riemann surfaces and minimal submanifolds, Ann. Scuola Norm. Sup. Pisa Cl. Sci. (4) 7 (1980), no. 1, 55-63. MR577325

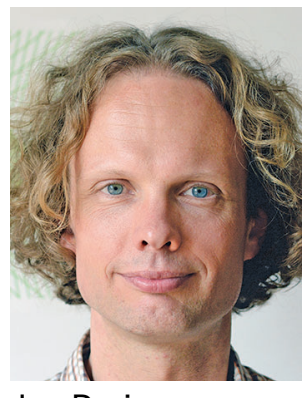

Jan Draisma

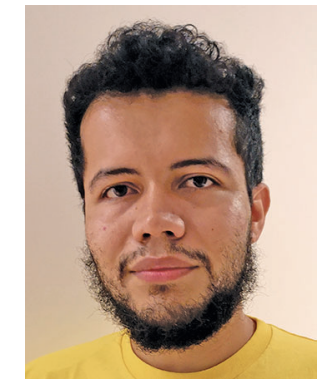

Alejandro Vargas
Credits

All article images are courtesy of the authors.

Photo of Jan Draisma is courtesy of Ivo Schmucki/University of Bern.

Photo of Alejandro Vargas is courtesy of Margarita Del Milagro López Aguilar. 